\title{
Electron Irradiation of Carbon Nanotubes: A Credible Defect Generation Technique to Improve Hydrogen Storage for Future
}

\author{
A. K. M. Fazle Kibria \\ Chemistry Division, Atomic Energy Centre, P. O. Box. 164, Ramna, Dhaka-1000, Bangladesh
}

\begin{abstract}
Electron irradiation technique was employed to induce atomic-scale defects in the walls of multi-walled carbon nanotubes (MWCNTs) using electron beam of energy $120 \mathrm{keV}$ and dose of $2.16 \times 10^{17}$ electrons $\mathrm{cm}^{-2} \mathrm{~s}^{-1}$. Structural transformations of the tubes attributable to irradiation exposure were monitored by high-resolution transmission electron microscope (HRTEM) and recorded by the connected CCD camera. It was observed that only five seconds (5s) of irradiation could shrink MWCNTs of diameter 15 nm having 11 graphitic layers towards the interior hole up to $2.2 \mathrm{~nm}$ and bend the tube. Irradiation incorporated vacancies and the topological defects generated on the tube walls were responsible for it. It was found that the electron irradiation could destroy such MWCNTs within 9 minutes. It was speculated that irradiation induced vacancies and their adjacent dangling bonds (DBs), the pentagon and octagon rings originated from the transformations of vacancies are highly influential to enhance the hydrogen storage in MWCNTs. From the time dependent HRTEM observations, it was found out that electron irradiation period less than 4 minutes is the optimum exposure time to achieve benefit of hydrogen storage in the MWCNTs as used in the present investigation. Electron irradiation induced defects could be used to enhance hydrogen storage in MWCNTs for future applications.
\end{abstract}

Keywords: Carbon nanotube, Electron irradiation, Defect, Vacancy, Hydrogen storage.

\section{Introduction}

It has been reported (Chen et al. 1999, Ye et al. 1999) that Carbon nanotubes (CNTs) could adsorb a great deal of hydrogen. Because of the necessity of such an efficient hydrogen storage material, the reports have produced a great excitement in the energy storage field. But the exciting observations were then found conflicting and compromised by the presence of moisture in the reactant, impurities in the CNTs, insufficient characterization of the tubes and thereby large errors in the hydrogen storage calculations (Yang, 2000, Ströbel et al. 2006). However, it was established that CNTs could store hydrogen at least $2 \mathrm{wt}$ \% which is comparable to that of conventional hydrogen absorbents. Investigators still believes that by modifying the nano-structured environments of CNTs, it might be possible to afford the USA department of Energy (DOE) set target hydrogen storage capacity of $6.5 \mathrm{wt} \%$ for vehicular transportations (Ströbel et al. 2006). It is notable that hydrogen is considered to be an environmentally friendly future fuel. Use of hydrogen in transportation system is one of the subjects to decrease the green house effect.

\footnotetext{
* Corresponding author: E-mail: kibriaf@yahoo.com
}

Hydrogen adsorption sites in CNTs reported so far are outside and inside of the tubes, between the tubes in bundles and ropes of single-wall carbon nanotubes (SWCNTs), and between the graphene layers in multi-wall carbon nanotubes (MWCNTs). Nowadays, it is well established that hydrogen storage capacity of CNTs depends on many parameters including their pretreatment, types, structural modifications, etc. Recently various attempts have been taken to boost up the hydrogen storage capacity of CNTs. One of the attempts seems to be highly influential and it is the generation of appreciable defects in the tube walls. Defects generated in CNTs by $\mathrm{KOH}$ (Chen and Huang 2007) and ammonia (Lin et al. 2009) treatments and hydrogen (Mu et al. 2006) and oxygen (Felten et al. 2009) plasma etchings have kept a great role in hydrogen storage enhancement. The defects not only helped to enrich hydrogen accesses into the interlayers and hollow interior of CNTs found also kept a significant role in promoting hydrogen spillover which is emerging one of the potential ways to enhance hydrogen storage capacity of CNTs (Zacharia et al. 2005). However, the increase in 
defects in graphitized layers of CNTs should be limited or their structure would be destroyed which might lead to lowering of interaction or potential energy between the hydrogen molecules and carbon atoms.

It has been reported that electron irradiation can easily induce defects in the wall of SWCNT (Hashimoto et al. 2004, Suzuki and Kobayashi 2006). Irradiated CNTs have been opening their new application possibilities (Kim et al. 2004, Wang et al. 2004, Gomez-navarro et al. 2005, Gupta et al. 2007). Irradiation induced defects are directly contributing to bring CNTs in practical applications. Generation of defects causes the structural transformation of CNTs. It has been observed that non-optimized electron irradiation severely destroy the graphitic network of CNTs (Ajayan et al. 1998, Banhart et al. 2005) which is not desirable for application purposes. It signifies that optimization of the irradiation state is a prerequisite condition for the engineering processing of CNTs. In order to control the structural transformations of CNTs, it is important to clearly understand the defect production mechanism, to realize the relationship of the energetic particle and exposure time with the diameter of CNTs and overall the extent of defect and their utilization arena. Understanding of the interaction of energetic particles with CNTs should minimize the damage of the tubes as it is an undesirable side effect. Because of the presence of curvature-induced strain in the atomic network of CNTs walls, the damage formation in them is quite different from that usually take place in the most common solids (Banhart et al. 2005).

The present paper has attempted to find out an optimum condition for the generation of defects in the walls of MWCNTs using energetic electron. The electron irradiation induced defect generation mechanisms along with the application possibility of the defects for the enhancement of hydrogen storage are extensively discussed.

\section{Materials and Methods}

MWCNTs synthesized by chemical vapor deposition (CVD) technique was purified by adopting the recognized purification method (Kibria et al. 2004) and was used in this study. At first, a suspension of purified MWCNTs was made in ethanol. The suspension was then sonicated for 20 minutes. To make sample for the transmission electron microscope (TEM) study, one drop of the solution was kept onto a carbon coated holey copper grid and dried in air at room tem- perature. Mild electron beam of energy $120 \mathrm{keV}$ and dose of $2.16 \times 10^{17}$ electrons $\mathrm{cm}^{-2} \mathrm{~s}^{-1}$ were applied directly on MWCNTs from the TEM at room temperature. The incident electron beam was capable to irradiate an area of $0.14 \mu^{2}$. Images of the irradiated MWCNTs were taken by a CCDcamera connected with the TEM. High-resolution TEM (HRTEM) images were recorded up to the destruction, i.e., amorphization of the MWCNTs.

\section{Results and Discussion}

Figure 1 shows the TEM image of MWCNTs sample prepared for the electron irradiation investigations. It can be seen that the sample consists of tubes of diameters about 10$22 \mathrm{~nm}$ but that of 14-16 nm diameters are higher in number. For this reason, the present study has attempted to focus mainly on the most populated MWCNTs. Effect of electron irradiation on tens of perfect MWCNTs were investigated. In each case, similar effects of irradiation were observed. The tubes having initial growth defects showed very high sensitivity to irradiation than the perfect tubes. The observed irradiation effect on a perfect tube is described below. The MWCNT consisted of 11 graphitic shells and its outer and inner diameters were $15.07 \mathrm{~nm}$ and $8.27 \mathrm{~nm}$, respectively.

Figure 2(a-f) are the HRTEM images of the electron irradiated MWCNT with respect to the exposure time of irradiation. From the Figure 2(a), it may be seen that within 5 s of irradiation, the walls of the tube showed a mode of shrinking towards the interior hole. Moreover, the appearance of a bending mode is clear at a place of the tube marked by a circle. The graphitic interlayer distances seem retained equal to that of non-irradiated tube which is $0.34 \mathrm{~nm}$. It can be seen that the tube shrinkage value is different in different places but showed a clear decreasing tendency towards the bending mode. Compared to the diameter of the non-irradiated tube, the shrinkage value in the imaged area towards the bending mode is about $1.30 \mathrm{~nm}-2.24 \mathrm{~nm}$.

From the HRTEM image shown in figure 2(b), it can be seen that the tube shrinkage value remained almost unchanged after 32s of irradiation. Within this period of irradiation, the bending angle reached to a value of about $12^{\circ}$. No noticeable discreetness of the graphitic layers is observed. From the figure 2(c), it may be seen that on continuation of irradiation up to 4 minutes, the tube diameter, diameter of the interior hole and the bending angle remained almost unchanged. The graphitic walls showed a good tolerance of electron irradiation. 


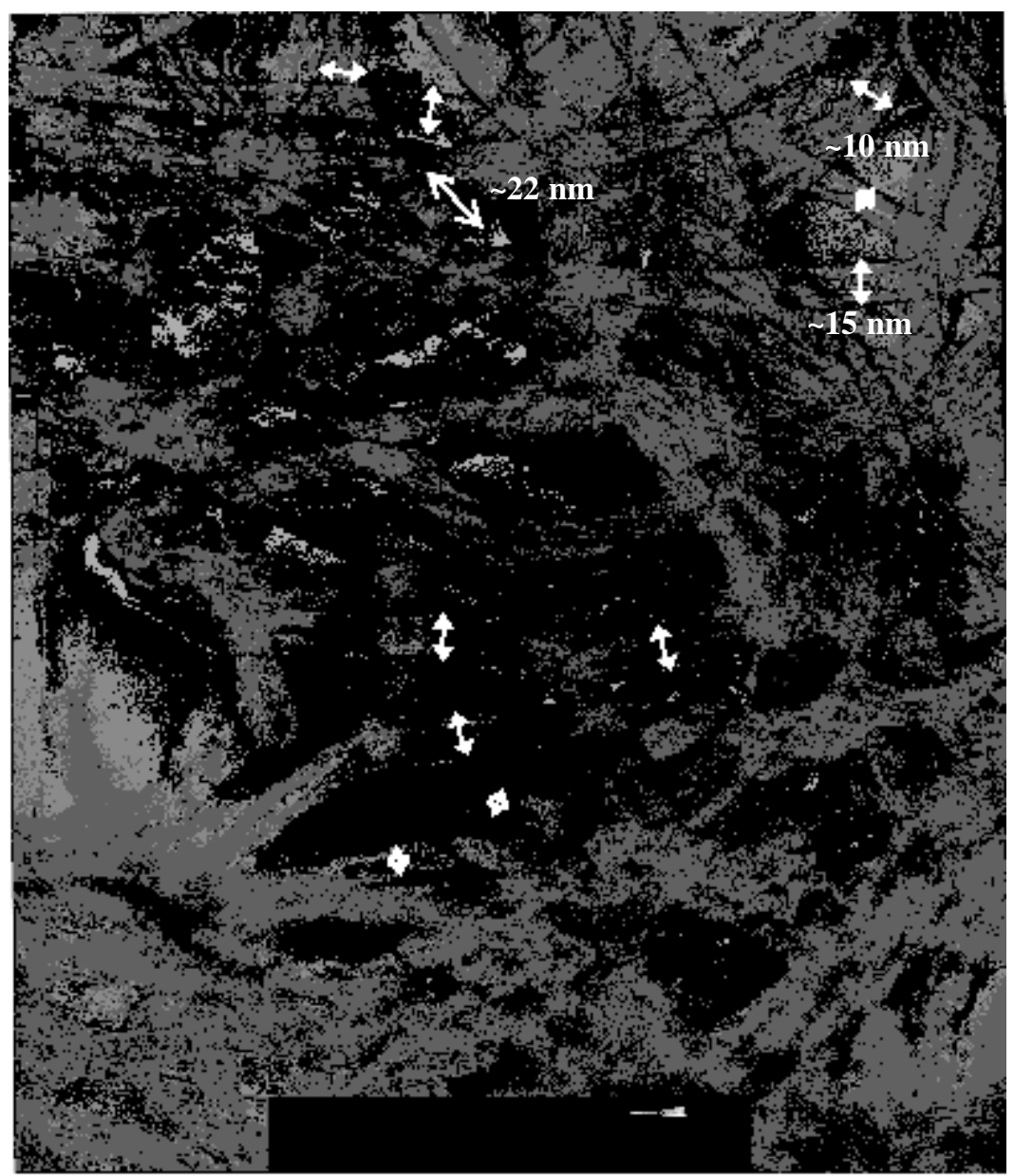

Fig. 1: Transmission electron microscopy (TEM) image of the multi-walled carbon naotubes (MWNTs) sample prepared for electron irradiation investigations.

It is observed that within this period of irradiation, small deposits of knocked-on carbon atoms from the graphitic layers hold the positions inside the layers and also the interior hole of the tube. Increase in irradiation time further one minute is showing understandable destruction of graphene layers in some places as presented by circle marks in the figure 2(d). A noticeable decrease in tube diameter and that of the interior hole also observed. On continuation of irradiation up to 6 minutes, the destruction of graphitic layers became severe. The tube walls were collapsed and transformed to amorphous like as shown in the figure 2(e). Both the diameters of the tube and the tube hole decreased remarkably. On further exposure to irradiation, the whole tube structured to amorphous like and the diameter showed a rapid decreasing trend due to the spattering of carbon atoms outwards as presented in figure 2(f).
Shrinking and bending of the MWCNT are informing the generation of electron irradiation induced defects in its walls and the defects are mostly vacancies (Hashimoto et al. 2004, Ajayan et al. 1998). The origin of vacancies is the displacement of $\mathrm{C}$-atoms from the hexagonal network of the graphitic walls by the energetic incident electrons. It is notable that the minimum incident electron energy of $86 \mathrm{keV}$ is required to remove a $\mathrm{C}$-atom from a graphitic wall by knock-on collision. At energies exceeding $139 \mathrm{keV}$, every carbon atom on a SWCNT is reported to be susceptible to ballistic ejection (Smith and Luzzi 2001). If the energy of the knocked-on C-atom is low, it can adsorb onto the nanotube wall as adatom. But in case of higher energy, the C-atom may spatter from the nanotube surface. The loss of Catom(s) from the hexagons of nanotube walls destroys the stable $s p^{2}$ hybridization of the neighboring C-atoms. The 

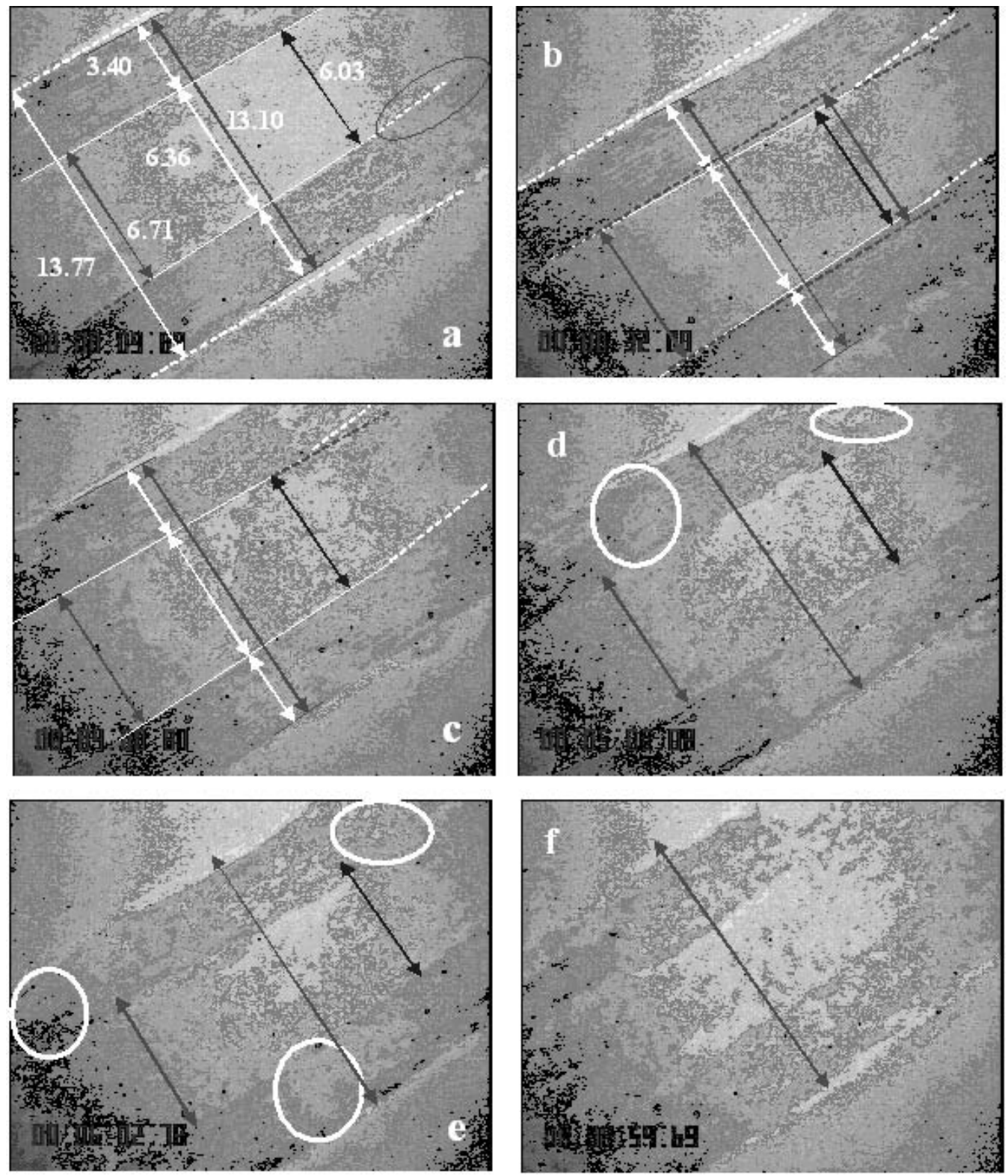

Fig. 2: High resolution transmission electron microscopy (HRTEM) images of the electron irradiated MWNT with respect to the exposure time of irradiation: (a) 5 seconds, (b) 32 seconds, (c) 4 minutes, (d) 5 minutes, (e) 6 minutes and (f) 9 minutes, respectively.

scenario of such structural transformation is presented in figure 3. From the figure 3(a), it may be seen that each sp type carbon atom consists of a dangling bond (DB). The dangling bonds (DBs) are very reactive to foreign atoms like hydrogen (Lu and Pan 2005). At this stage, the new sp type hybridized C-atoms may serve as the suitable sites to make bond with the incoming hydrogen atoms to enhance the hydrogen storage in CNTs. It may be seen that a single vacancy (SV) and a double vacancy (DV) can easily adsorb three and four hydrogen atoms, respectively. The possibility of molecular hydrogen adsorption by the dangling bonds can also be considered. These vacancies are the simple defects of graphitic walls (Hashimoto et al. 2004, Ajayan et al. 1998). 
Due to the formation of vacancies, the nanotube walls practically suffer from higher strain than the unique state because of having curvatures. In case of SWCNT, the strain is very high. With increasing the diameter of tubes, the strain gradually decreases. For MWCNT, the innermost wall suffers the highest strain (Banhart et al. 2005). To relief the excess strain, the vacancies can be transformed to other types of defects known as topological defects (Hashimoto et al. 2004, Ajayan et al. 1998). The scenario of the potential transformation of vacancies to topological defects is presented in the figure 3(b). It may be seen that two of the three DBs of a SV may recombine each other to form a pentagon ring with a DB left and transforming to a so-called pentagon-1DB (51DB) defect. In case of a DV, two and two of DBs separately may combine each other to form two pentagons and an octagon ring to generate a pentagon-octagon-pentagon (5-85) defect. On the other hand, the SV may transform to another type of defect known as Stone-Wall defect (SWD). This defect originates by the rotation of one of the $\mathrm{C}-\mathrm{C}$ bonds in $\pi / 2$, resulting in the transformation of four nearby hexagons into a pair of heptagons having a common side and the separation of two pentagons as shown in figure 3(c). It is commonly represented by 5-7-7-5 defect. The configuration of this defect has a 5-7 core attached to an inverted 7-5 core. They can be separated into two 5-7 defects and may propagate easily. This propagation corresponds to a plastic flow of defects on the graphitic wall of CNTs (Ajayan et al. 1998). Recently, it has been experimentally proven that defects in a graphene layer such as vacancies and adatoms, and topological defects are numerous and stable under controlled electron irradiation (Hashimoto et al. 2004). Transformations of the primary vacancies to the topological defects as described above are responsible for the observed shrinking and bending of the irradiated MWCNTs.

In case of a 5-1DB defect (figure 3b), the DB can easily adsorb atomic or molecular hydrogen. The pentagon ring may also be influential to adsorb hydrogen by breaking one of its $\mathrm{C}-\mathrm{C}$ bond systems and rearranging the $\mathrm{DB}$ site in order to reach in more stable state. The voids of 5,7 and 8 membered rings presents in the defects may incorporate hydrogen through them in the graphitic interlayers and the interior hole of MWCNTs. Recently, authors (Lu and Pan 2005) have carried out a simulation study on the interaction of hydrogen with the SV and DV defects present in CNTs. It was observed that atomic hydrogen can chemically adsorb on the DB of 5-1DB defect without any energy barrier, but a hydrogen molecule may adsorb on the DB site by overcoming an amicable energy barrier of no more than about $1.6 \mathrm{eV}$. When the DB is saturated by a single hydrogen atom, the local structure around the saturated dangling bond atom might exhibit metastable geometries or a lowest energy geometry, in which the metastable structures feature $\mathrm{sp}^{3}$-like hybridization and the lowest energy structure shows $\mathrm{sp}^{2}$-like hybridization. It was shown that neither atomic hydrogen nor hydrogen molecule in the outer surface of the tube can enter the inner space via the pentagon void of 5-1DB, whereas they can enter via the larger space having in 5-8-5 defects. The eight-membered ring of this defect offers a large void which can easily serve as an access point for hydrogen to traverse between the outer and inner sides of graphitic layers. It was strongly suggested that hydrogen leakage and fill-in for the defected CNTs containing DVs or defects supplying larger voids in size should be taken into account when the tubes serve as a storage cell for hydrogen molecules. The study informs the high possibility of penetration of atomic and molecular hydrogen in the graphitic interlayers of irradiated MWCNTs.

Presently observed shrinking and bending of MWCNTs during irradiation is also clearly indicating their damage. Actually, what happened when the MWNTs were subjected to chemical treatment and plasma etching? It is notable that the chemically treated tubes stored hydrogen up to 4.5wt.\%. The shown HRTEM image of $\mathrm{KOH}$ treated MWNT in the report is a highly damaged tube (Chen and Huang, 2007). Moreover, the tube has no interior hole, the room for excess hydrogen storage, which is an unlike feature than the common MWCNTs. The graphitic layers were broken in different places. The places were marked as defects by the investigators. The defective places may have a lot of dangling bonds, the bonding sites for incoming hydrogen to enhance hydrogen storage. The increase in hydrogen storage by these tubes is indicating that a fraction of the stored hydrogen entered into the graphitic interlayers and it was claimed by the authors too. However, the stability of such defective tubes may be a question during the adsorption-desorption cycling. The HRTEM image of the plasma etched MWCNT presented in the report shows that about $50 \%$ of the graphitic layers starting from the outermost wall of the tube were damaged during the processing (Mu et al. 2006). The dangling bonds present in the damaged areas probably contributed in the hydrogen storage enhancement up to $1.4 \mathrm{wt}$ \%. From the above discussions, it can be realized that the extent of damage of MWCNTs by chemical treatment, plasma etching and electron irradiation are different. In case of electron irradiation, the damage is mostly atomistic. In the present experimental setup, the damage of MWCNTs seems not so severe before the 4 minutes of irradiation exposure. Up to 4 minutes of irradiation, the state of the MWNT seems to be far better than those of chemical and plasma treated MWNTs (Chen and Huang 2007, Mu et al. 2006). From this comprehensive 


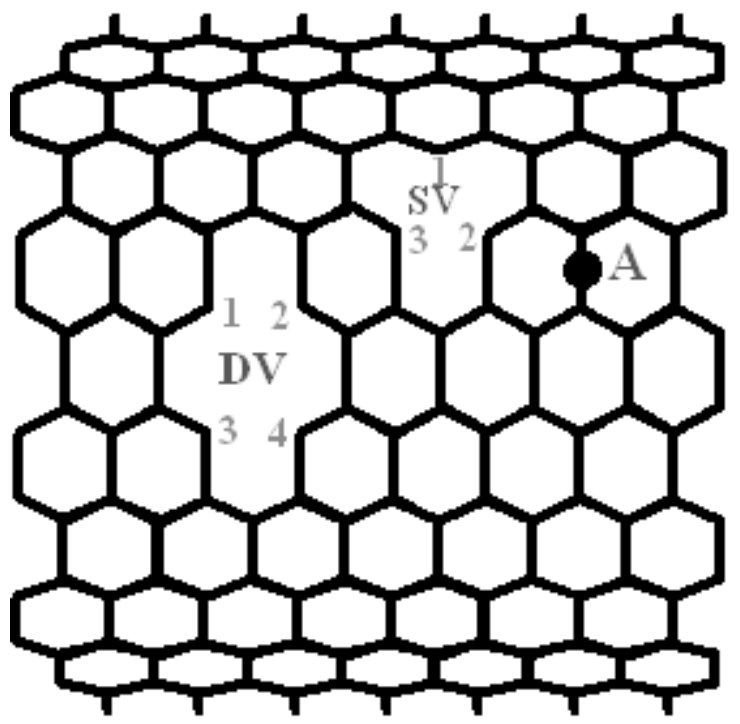

(a)

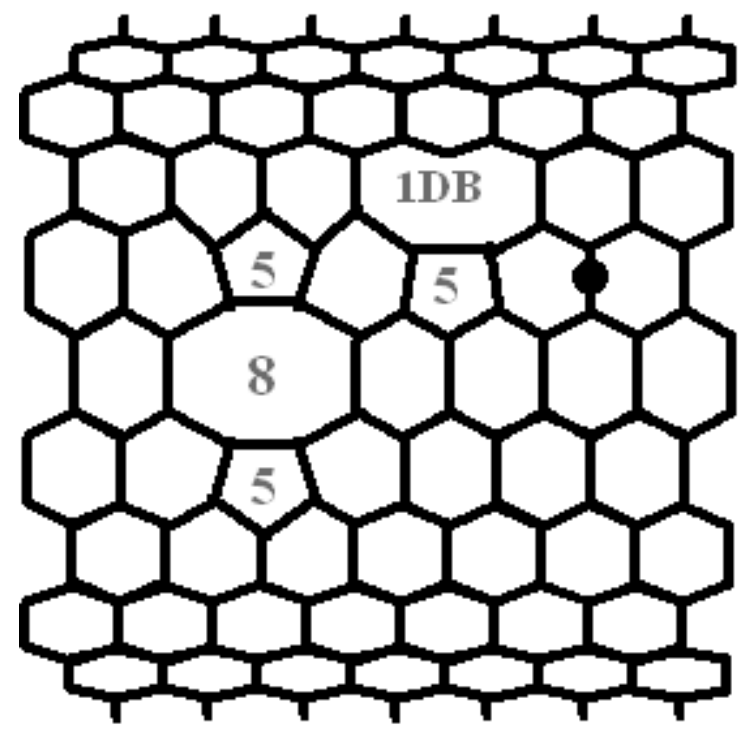

(b)

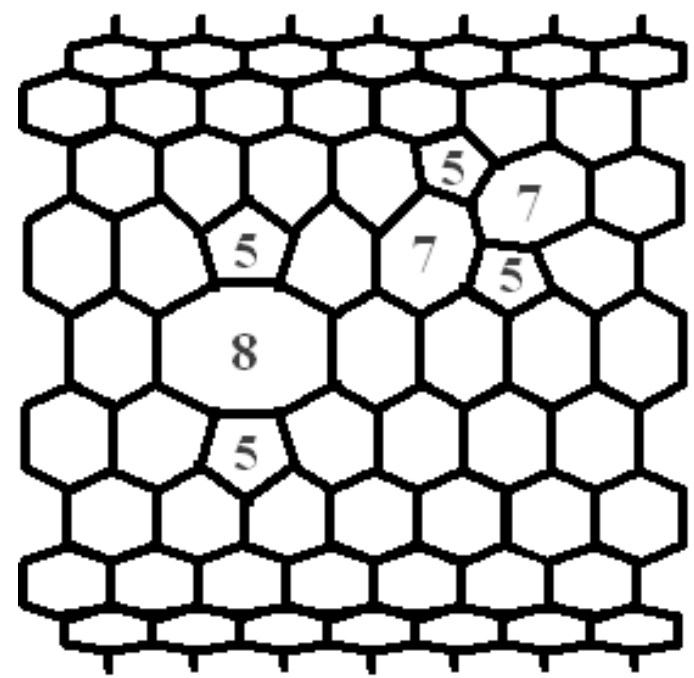

(c)

Fig. 3: Mechanisms of the generation of electron irradiation induced vacancies and topological defects in the walls of MWNTs: (a) Generation of single vacancy (SV), double vacancy (DV) and adatom (A); (b) Generation of pentagon-dangling bond (5-1DB) and pentagon-octagon-pentagon (5-8-5) defects from SV and DV; and (c) Generation of pentagon-heptagon (5-7) stone-wall (SW) defects with other defects.

findings, it may be concluded that controlled electron irradiation of MWCNTs could bring the best and efficient state for the enhancement of hydrogen adsorption in them than other available modification methods.

The appearance of small deposition of carbon in the graphitic interlayers and the interior hole of the tube after 4 minutes of irradiation (Fig. 2c) is indicating that the incident electrons are energetic enough to knock-on carbon atoms from all the graphitic layers of the MWCNT. The existence of all the starting 11 graphitic layers after 5 minutes of irradiation, while small areas of walls were destroyed (Fig. 2d), is not supplying any information whether the MWCNT began to destroy from the innermost shell or the outermost shell. Authors (Banhart et al. 2005) have reported the destruction of a MWCNT having diameter of about $7.85 \mathrm{~nm}$ (hole diameter $\sim 4.45 \mathrm{~nm}$ ) and 6 shells under electron beam of diameter $15 \mathrm{~nm}$ and beam current density of $450 \mathrm{~A} / \mathrm{cm}^{2}$. The diameter of the tube is only $52 \%$ of the presently used tube. It is notable that such a defective and destructed nan- 
otube or the destructed places can never be beneficial for hydrogen storage. The destruction found begins severely from the inner shell of the tube. The severances of destruction can be realized from the application of $1.3 \times 10^{4}$ folds higher beam current densities over 14 times lower areas of MWCNT than the present case. It may be taken as an indication that by controlling the electron irradiation condition, the destruction of MWCNT, i.e., the extent of defect in MWCNTs can be controlled. Authors (Hashimoto et al. 2004) have used a low energetic electron beam $120 \mathrm{keV}$ and dose of $6 \times 10^{18} \mathrm{e} \mathrm{cm}^{-2} \mathrm{~s}^{-1}$ which corresponds to the beam intensity in the range of $\mathrm{mA} / \mathrm{cm}^{2}$. The study successfully observed the primary and secondary defects on SWCNT wall which are desirable for the hydrogen storage. Presently used electron dose is more nominal and it is about 28 times lower than that used by the authors (Hashimoto et al. 2004).

Irradiation of SWNCTs with $\mathrm{Ar}^{+}$beam has reported (Krasheninnikov et al. 2001) that SV and vacancy-related defects are the most prolific defects as appeared during the electron beam irradiation (Hashimoto et al. 2004). Authors (Khare et al. 2003) have evidenced by FT-IR and UV-visNIR measurements that proton irradiation has resulted $\mathrm{C}-\mathrm{H}$ bonds in SWCNTs. Authors (McDaniel et al. 2006) have also reported the $\mathrm{C}-\mathrm{H}$ bond formation in the proton irradiated SWCNTs where implanted hydrogen was estimated by elastic recoil detection (ERD) measurements with energetic $\mathrm{He}^{+}$beam. Recently, authors (McDaniel et al. 2007) have reported that introduction of defects on the SWCNTs by $\mathrm{H}^{+}$ irradiation offered an alternative method to activate hydrogen adsorption and enhance the hydrogen storage in SWCNTs. It seems to be reasonable to expect the generation of defects in SWCNTs by energetic proton alike as those generates by the energetic electron. On analyzing the findings of the effects of electron irradiation on the imaged MWCNT (figures 2a-f), it can be realized that electron irradiation of such a nanotube up to a time period of less than 4 minutes is not so destructive one at the set experimental conditions. At this stage, the MWCNTs may contain a lot of defects and it is the best stage to utilize the electron irradiation induced defects for the hydrogen storage enhancement. It can be concluded that controlled electron irradiation of MWCNTs would help much to enhance the hydrogen storage in them for the future applications.

\section{Conclusion}

An attempt has been taken to generate atomic-scale defects on the walls of MWNTs by using electron irradiation technique. Depending on the highest population of the tubes in the sample, MWCNTs of inner diameter about $8 \mathrm{~nm}$ and graphitic shells about 10-12 in numbers were chosen for the irradiation investigations. An electron beam of energy 120 $\mathrm{keV}$ and dose of $2.16 \times 10^{17}$ electrons $\mathrm{cm}^{-2} \mathrm{~s}^{-1}$ was used for this purpose. During the irradiation treatment, the structural transformations of the tubes were monitored by high-resolution transmission electron microscope (HRTEM) and imaged by a CCD camera connected with it. From the taken HRTEM images of a tube, it was observed that within 5 seconds of irradiation it bended and shrunk about $2.3 \mathrm{~nm}$ towards the interior hole. On increase in irradiation time up to 4 minutes, the graphitic layers of the tube showed high tolerance of irradiation, i.e., further noticeable shrinking and bending was not observed. After then, the graphitic layers started to destroy. Complete destruction of MWCNTs observed after 9 minutes of irradiation. It was attributed that shrinking and bending of the MWCNTs occurred due to the generation of electron irradiation induced defects in their walls and the defects are mostly vacancies. The vacancies were originated from the displacement of C-atom(s) by the energetic electrons from the tube walls. Generation of vacancies imparted higher strain in the walls. In order to relief the excess strain, the vacancies might be transformed to other types of defects known as 5-1DB, 5-8-5 and 5-7-7-5 defects. It was speculated that the DBs present around the vacancies and there after in 5-1DB defects, the 5-1DB itself and the 8 membered ring present in the 5-8-5 defect are highly influential in hydrogen adsorption. The 5-1DB defect is capable to adsorb atomic and molecular hydrogen. On the other hand, 8 membered ring of 5-8-5 defect is capable to traverse atomic and even molecular hydrogen from one side of the wall to other side. It means that nanotube walls after irradiation are achieving some extra quality to adsrob a lot of hydrogen in them. From the time dependent HRTEM observations, it was found out that electron irradiation time about 4 minutes is the optimum exposure time to achieve benefit of hydrogen storage in the MWCNTs as used in the present study. The study is expecting that the electron irradiation induced defects would highly contribute to enhance hydrogen storage in MWCNTs for the future applications. The feasibility of the improvement of hydrogen storage in the MWCNTs is highly discussed through the defect generation mechanisms. In order to deliver comprehensive information, the attempts have been taken so far to enhance the hydrogen storage in MWCNTs by incorporating defects through the chemical treatment and plasma etching were also discussed.

\section{References}

Ajayan P. M., Ravikumar V. and Charlier J. C. (1998). Surface reconstructions and dimensional changes in single-walled carbon nanotubes. Phys. Rev. Lett. 81: 1437-1440. 
Banhart F., Li J. X. and Krasheninnikov A. V. (2005). Carbon nanotubes under electron irradiation: stability of the tubes and their action as pipes for atom transport. Phys. Rev. B 71: 241408-4.

Chen C. H. and Huang C. C. (2007). Hydrogen storage by $\mathrm{KOH}$-modified multi-walled carbon nanotubes. Int. J. Hydrogen Energy 32: 237-246.

Chen P., Wu X., Lin J. and Tan K. L. (1999). High $\mathrm{H}_{2}$ uptake by alkali-doped carbon nanotubes under ambient pressure and moderate temperatures. Science 285: 91-93.

Felten A., Ghijsen J., Pireaux J. J., Drube W., Johnson R. L., Liang D., Hecq M., Tendeloo G. V. and Bittencourt C. (2009). Electronic structure of Pd nanoparticles on carbon nanotubes. Micron 40: 74-79.

Gomez-navarro C., Pablo P. J. de Gomez-Herrer J., Biel B., Garcia-Vidal F. J., Rubi A. and Flores F. (2005). Tuning the conductance of single-walled carbon nanotubes by ion irradiation in the Anderson localization regime. Nat. Mater. 4: 534-539.

Gupta S., Patel R. J., Smith N., Giedd R. E. and Hui D. (2007) Room temperature dc electrical conductivity studies of electron-beam irradiated carbon nanotubes. Diam. Relat. Mat. 16: 236-242.

Hashimoto A., Suenaga K., Gloter A., Urita K. and Iijima S. (2004) Direct evidence for atomic defects in graphene layers. Nature 430: 870-873.

Khare B., Meyyappan M., Moore M. H., Wilhite P., Imanaka H. and Chen B. (2003). Proton irradiation of carbon nanotubes. Nano Lett. 3: 643-646.

Kibria A. K. M. F., Shajahan Md. Mo Y. H., Kim M. J. and Nahm K. S. (2004). Long activity of Co-Mo/MgO catalyst for the synthesis of carbon nanotubes in largescale and application feasibility of the grown tubes. Diam. Rela. Mat. 13:1865-1872.

Kim D. H., Kim C. D. and Lee H. R. (2004). Effects of the ion irradiation of screen-printed carbon nanotubes for use in field emission display applications. Carbon 42: 1807-1812.

Lin K. Y., Chang J. K., Chen C. Y. and Tsai W. T. (2009). Effects of heat treatment on materials characteristics and hydrogen storage capability of multi-wall carbon nanotubes. Diam. Relat. Mat. 18: 553-556.
Lu A. J. and Pan B. C. (2005). Interaction of hydrogen with vacancies in a $(12,0)$ carbon nanotube. Phys. Rev. B 71: 165416-6.

Mu S. C., Tang H. L., Qian S. H., Pan M. and Yuan R .Z. (2006) Hydrogen storage in carbon nanotubes modified by microwave plasma etching and Pd decoration. Carbon 44: 762-767.

McDaniel F. D., Holland O., W. Naab F. U., Mitchell L. J., Dhoubhadel M. and Duggan J.L. (2006). Ion beam analysis of hydrogen retained in carbon nanotubes and carbon. Nucl. Inst. Met. Phys. Res. B 249: 330-334.

McDaniel F. D., Naab F. U., Holland O. W., Dhoubhadel M. Mitchell L. J. and Duggan J. L. (2007). Low-energy ion irradiation effects on hydrogen absorption and desorption in carbon nanotubes. Surf. Coat. Tech. 201: 8564-8567.

Smith B. W. and Luzzi D. E. (2001). Electron irradiation effects in single wall carbon nanotubes. J. Appl. Phys. 90: 3509-3512.

Ströbel R., Garche J., Moseley P. T. , Jörissen L. and Wolf G. (2006) Hydrogen storage by carbon materials. $J$. Power Sourc. 159: 781-801.

Suzuki S. and Kobayashi Y. (2006). Diameter dependence of low-energy electron and photon irradiation damage in single-walled carbon nanotubes. Chem. Phys. Let. 430: 370-374.

Wang Z., Yu L.. Zhang W. , Ding Y. Li, Y., Han J., Zhu Z., Xu H., He G., Chen Y. and Hu G. (2004). Amorphous molecular junctions produced by ion irradiation on carbon nanotubes. Phys. Lett. A 324: 321-325.

Yang R. T. (2000). Hydrogen storage by alkali-doped carbon nanotubes-revisited. Carbon 38:623-626.

Ye Y., Ahn C. C., Witham C., Fultz B., Liu J., Rinzler A. G., Colbert D., Smith K. A. and Smalley R. E. (1999). Hydrogen adsorption and cohesive energy of singlewalled carbon nanotubes. Appl. Phys. Lett. 74: 23072309.

Zacharia R., Kim. K. Y. Kibria A. K. M. F. and Nahm K. S. (2005). Enhancement of hydrogen storage capacity of carbon nanotubes via spill-over from vanadium and palladium nanoparticles. Chem. Phys. Lett. 412: 369-75.

Received : January 13, 2009;

Accepted : March 16, 2010 